\title{
PLAGL1 protein is differentially expressed in the nephron segments and collecting ducts in human kidney
}

\author{
Janusz Godlewski', Bartlomiej E. Krazinski' ${ }^{1}$, Jacek Kiezun ${ }^{1}$, Przemyslaw Kwiatkowski ${ }^{1}$, \\ Marian Sulik ${ }^{2}$, Michal Tenderenda ${ }^{3}$, Wojciech Biernat ${ }^{4}$, Zbigniew Kmiec ${ }^{1,5}$ \\ ${ }^{1}$ Department of Human Histology and Embryology, Faculty of Medical Sciences, University of Warmia \\ and Mazury in Olsztyn, Poland \\ ${ }^{2}$ Department of Pathomorphology, Faculty of Medical Sciences, University of Warmia and Mazury \\ in Olsztyn, Poland
}

${ }^{3}$ Department of General and Oncological Surgery, Faculty of Medical Sciences, University of Warmia and Mazury in Olsztyn, Poland

${ }^{4}$ Department of Pathomorphology, Medical University of Gdansk, Poland

${ }^{5}$ Department of Histology, Medical University of Gdansk, Poland

\begin{abstract}
Introduction. PLAGL1 (pleiomorphic adenoma gene-like 1) is a C2H2-type zinc finger transcription factor associated with the regulation of cell growth and development. Although PLAGL1 expression in kidney was assessed by biochemical methods, the exact localization of the PLAGL1 protein in human kidney has not yet been described.

Material and methods. Macroscopically unchanged specimens of kidney tissue were collected from 39 patients undergoing nephrectomy due to renal cell carcinoma. $\mathrm{H} \&$ E staining of paraffin sections was used to assess histology of the kidney whereas immunohistochemistry was used to localize PLAGL1 protein in kidney compartments. In addition, database sequences search for putative PLAGL1 binding sites among the kidney-related genes was performed.

Results. PLAGL1 staining intensity differed depending on the kidney compartment. Strong PLAGL1 immunoreactivity was found in thick ascending limbs of Henle's loop, distal tubules and collecting ducts, whereas PLAGL1 expression in proximal tubules and renal corpuscles (including podocytes) was moderate and weak, respectively. By the in sillico screening of promoter sequences for PLAGL1 specific DNA-binding sites GGGGCCCC we designated 43 candidate genes for PLAGL1-regulated genes. Analysis of their functional annotations identified three significantly over-represented gene sets: inositol phosphate metabolic processes (GO), endocrine and other factor-regulated calcium reabsorption (KEGG) and calcium signaling pathways (KEGG). Conclusion. Differences in the renal expression of PLAGL1 suggest that this protein may be involved in the regulation of several cellular pathways both as transcriptional factor and coactivator/corepressor of other transcription factors reflecting its role in the cell type-specific control of gene expression. (Folia Histochemica et Cytobiologica 2015, Vol. 53, No. 1, 96-104)
\end{abstract}

Key words: PLAGL1; Zac1; human kidney; proximal tubules; distal tubules; collecting ducts; IHC; in silico gene analysis 


\section{Introduction}

PLAGL1 is a C2H2-type zinc finger transcription factor, encoded by the PLAGL1 gene (pleiomorphic adenoma gene-like 1; known also as ZAC1 for Zinc -finger protein which regulates apoptosis and cell-cycle arrest or LOT1 for Lost-on-transformation 1). Many transcript variants encoding two main PLAGL1 isoforms (44.7 and $50.8 \mathrm{kDa}$ ) were found for this gene (Ensembl ENSG00000118495). PLAGL1 is ubiquitously expressed in a number of normal embryonic and adult tissues [1,2]. Studies performed so far allowed to determine PLAGL1 as a factor contributing to the regulation of cell growth and differentiation in several physiological and pathological processes. It was shown that $P L A G L 1^{-/-}$knock-out mice demonstrated fetal growth retardation, altered bone formation and neonatal mortality [3]. In the course of the embryonic development an increased PLAGL1 expression was detected in the nervous system, chondrogenic tissue, liver, myocardium and skeletal system [2]. The studies on the transient neonatal diabetes mellitus (TNDM) indicate the role of the PLAGL1 gene overexpression in etiopathogenesis of this disease [4,5].PLAGL1 was proposed as a putative tumor suppressor gene as it shares its anti-proliferative and pro-apoptotic activity with TP53, i.e. inhibits cell growth and promotes cell cycle arrest $[6,7]$. PLAGL1 gene deletion as well as decrease of its expression were found in many types of human neoplasms: breast and ovarian cancers, basocellular skin cancer and adrenal gland tumors $[1,2]$.

Previous studies reported that PLAGL1 binds DNA and can act as a transcription factor by sequence-specific DNA binding [1,8]. The consensus binding nucleotide sequence for PLAGL1 was identified as GGGGGGCCCC [1]. It was documented that PLAGL1 can improve DNA-binding activity of histone acetyltransferases, stabilize p53 and enhance p53-dependent gene expression [9-12]. PLAGL1 can also act as transcriptional coactivator or corepressor of hormone-dependent activity of nuclear receptors [12]. The latter can be supported by the fact that in adults PLAGL1 mRNA shows a high expression in most steroid-responsive tissues, including human kidney [1].

The aim of our study was to provide information about the localization of the PLAGL1 protein in normal human kidney using immunohistochemical (IHC) technique. We found much stronger PLAGL1 immunoreactivity in thick ascending limbs of Henle's loop, distal tubules and collecting ducts than in proximal tubules and podocytes. We sought to combine our experimental and in sillico data to suggest possible role of PLAGL1 in the functions of human kidney.

\section{Material and methods}

Patients. This study was approved by the Bioethical Commission of the University of Warmia and Mazury (permissions No. 4/2010 and 33/2010). A written agreement was obtained from each participant. Postoperative kidney samples were harvested from patients subjected to radical nephrectomy due to renal cell carcinoma who were operated on in the years 2010-2012 in Warmia and Mazury Oncological Center in Olsztyn, Poland. Specimens were obtained from 39 patients: 23 males and 16 females, aged between 31 and 83 years $(62.7 \pm 10.5$ years; mean \pm SD). Directly after the nephrectomy, specimens for routine histological evaluation and IHC staining were resected from macroscopically cancer-free part of the kidney and fixed in $4 \%$ buffered-formaldehyde ( $\mathrm{pH} 7.4$ ). Simultaneously, kidney tissue samples for Western blot analysis were frozen in liquid nitrogen and stored at $-80^{\circ} \mathrm{C}$.

Validation of anti-human PLAGL1 antibodies by Western immunoblotting. Prior to the IHC, the utility of primary antibody for staining procedure was validated using Western blotting technique. Homogenates were prepared from six macroscopically unchanged parts of kidneys and immunoblotting procedure using the rabbit monoclonal anti-human PLAGL1 antibody (1:1000; Anti-PLAGL1/ZAC antibody, \#ab129063, Abcam, Cambridge, UK) was performed according to the previously described procedure [13]. Homogenate of human tonsil was used as immunoblotting the positive control. Spectra Multicolor High Range Protein Ladder (Thermo Fisher Scientific, \#26625; Waltham, MA, USA) was used as a molecular mass marker.

Tissue sampling and immunohistochemistry. Kidney tissue specimens of 39 patients were processed in ethanol and xylene and embedded in paraffin. Seven $\mu \mathrm{m}$ thick tissue sections were cut, mounted on gelatin-coated slides, deparaffinized and rehydrated. Antigen retrieval was carried out using Epitope Retrieval Solution (Dako, Glostrup, Denmark) and microwaving (ca $100^{\circ} \mathrm{C}, 4$ times for $5 \mathrm{~min}$ ), cooled, and washed for $30 \mathrm{~min}$ in PBS, $\mathrm{pH}$ 7.4. Endogenous peroxidase activity was quenched by the incubation of sections in 3\% hydrogen peroxide solution in methanol for $10 \mathrm{~min}$. Then, the sections were washed in PBS and incubated for $30 \mathrm{~min}$ in $2.5 \%$ horse serum (Abcam) as a serum blocking procedure. Subsequently, primary antibody, rabbit monoclonal anti-human PLAGL1 (1:2000; Anti-PLAGL1/ /ZAC antibody, \#ab129063, Abcam), was applied. After overnight incubation at $4^{\circ} \mathrm{C}$, slides were washed in PBS and treated for 30 min with secondary antibody (ImmPRESS Universal reagent Mouse Anti-Rabbit Ig, Vector Laboratories, Burlingame, CA, USA). Then, slides were washed in PBS and developed in 3,3'-diaminobenzidine (Liquid DAB + Substrate Chromogen System, Dako). Sections 
were counterstained in Mayer's hematoxylin and washed in PBS. Slides were dehydrated in ethanol, cleared in xylene, embedded in DPX (Sigma Aldrich, St. Louis, MO, USA) and coverslipped. All sections were stained in duplicates. Negative controls (without primary antiserum) were run in parallel with antibody-incubated sections.

Evaluation of immunohistochemical reactions. All preparations were examined using Olympus BX-41 microscope and archived with Olympus XC50 digital camera using the Cell* software (Olympus, Tokyo, Japan). Hematoxylin and eosin (H \& E) staining was performed to confirm normal morphology of the kidney. PLAGL1 immunostaining intensity was scored in the renal corpuscles, epithelia of the proximal tubules, thick ascending limbs of Henle's loop, distal tubules and collecting ducts.

It was arbitrarily assumed that a very strong staining in distal tubules and collecting ducts reported in the kidney of patient No. 31 was scored for $100 \%$ and the other sections were scored in relation to the intensity of that staining. Sections stained by the immunohistochemical (IHC) method were classified (and scored) as: negative (score 0), trace (1-10\%; score 10$)$, weak (11-30\%; score 30$)$, moderate (31-60\%; score 60), strong (61-80\%; score 80$)$ and very strong (81-100\%; score 100) immunoreactivity.

Database sequences search for putative PLAGL1 binding sites. BioMart data-mining tool (BioMart; http://www.biomart.org/) [14] was used to retrieve the sequences located 2-kb upstream the transcription start site (TSS) of 1054 transcripts belonging to 478 genes which were found to be associated with the kidney physiology on the basis of annotations available at KEGG database (Kyoto Encyclopedia of Genes and Genomes; http://www.genome.jp/kegg) [15] and The Human Proteome database (Human Protein Atlas; http://www.proteinatlas.org/humanproteome/kidney) [16]. The sequences were sought for an alignment (only exact matches) with the most conservative core of PLAGL1 consensus nucleotide sequence: GGGGCCCC [1]. ConsensusPathDB-human (CPDB; http://cpdb.molgen. mpg.de/) [17] was used to identify the association of putative PLAGL1 target genes with kidney-related pathway-based sets. Hypergeometric distribution-based over-representation analysis was performed using a list of queried genes as a background. Matches with FDR corrected $p$-values $<0.05$ were considered as significantly over-represented.

Statistical analysis. Statistical analyses were performed using Prism 6.04 software (GraphPad software Inc., La Jolla, CA, USA). Normal distribution was confirmed by D'Agostino \& Pearson omnibus test. One-Way ANOVA followed by Tukey's multiple comparisons test was used to statistically assess differences of PLAGL1 immunostaining intensity.

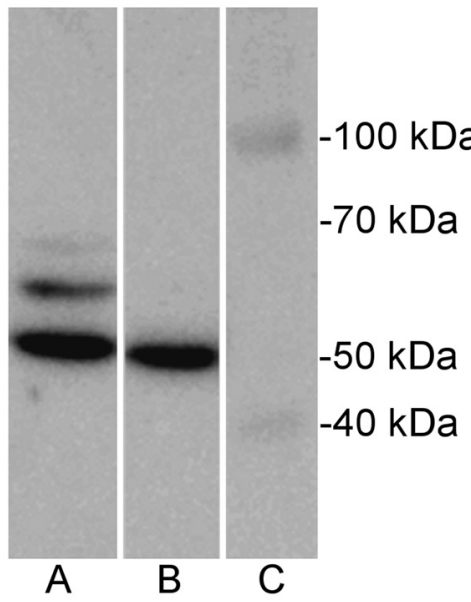

Figure 1. Representative Western blot showing PLAGL1 expression in human kidney homogenate (lane B). Lane A — positive control - human tonsil; lane $\mathrm{C}$ - molecular mass marker Spectra Multicolor High Range Protein Ladder, Thermo Fisher Scientific \#26625 (Waltham, MA, USA)

$P$-values $<0.05$ were considered as statistically significant. Pearson correlation, Mann-Whitney and Kruskal-Wallis tests were used to analyze the correlations between experimental results and clinicopathological data of the patients.

\section{Results}

\section{PLAGL1 immunoblotting}

All tested kidney homogenates $(\mathrm{N}=6)$ were positive for PLAGL1 protein exhibiting a strong band of PLAGL1-predicted size (approximately $51 \mathrm{kDa}$; Figure 1). Additional bands were present in samples used as a positive control, human tonsil, while in kidney homogenates additional bands were only barely visible.

\section{PLAGL1 immunohistochemistry}

The H \& E staining confirmed a normal morphology of cortex and medulla in the examined parts of human kidneys (Figure 2A). PLAGL1 expression in kidney was examined by IHC staining. PLAGL1 immunoreactivity was found in the cortex and medulla of 38/39 (97.4\%) of the tested kidneys (Figure 2B). PLAGL1 expression was noted in the epithelial cells of the parietal layer of Bowman's capsule, podocytes, mesangial cells and the epithelia lining proximal tubules, thick ascending limbs of Henle's loop, distal tubules, and collecting ducts (Figures 3 and 4). Only a weak immunoreactivity was found in some but not all nuclei of thin limbs of Henle's loop cells while no reaction was 

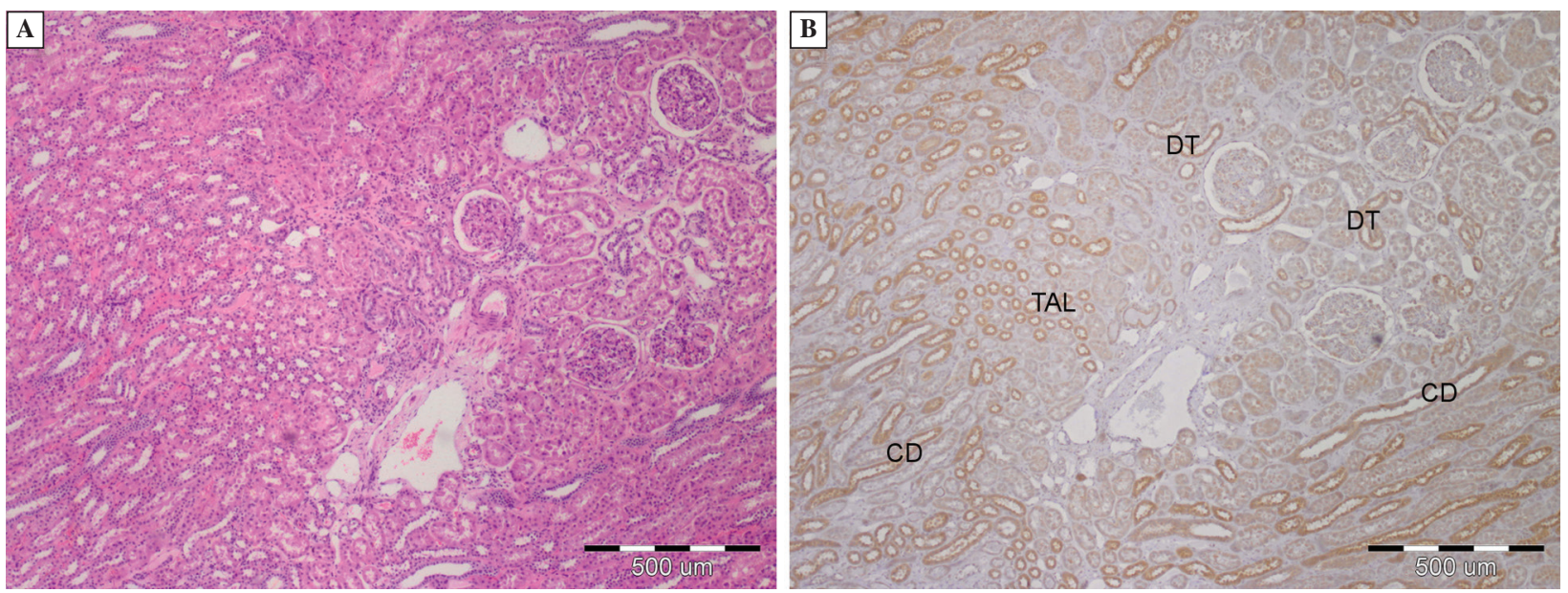

Figure 2. Structure of the normal human kidney revealed by (A) H \& E staining and (B) PLAGL1 immunoreactivity. A. Normal histology of kidney cortex and medulla; B. Strong PLAGL1 immunoreactivity is evident in thick ascending limbs (TAL) of Henle's loop in the outer stripe of the outer medulla, distal tubules (DT) in the kidney cortex and collecting ducts (CD) both in the cortex and medulla. Moderately intense PLAGL1 immunoreactivity is visible in proximal tubules and within the renal corpuscles. Immunohistochemical (IHC) staining was performed as described in Methods. Total magnification $\times 40$; scale bar, $500 \mu \mathrm{m}$
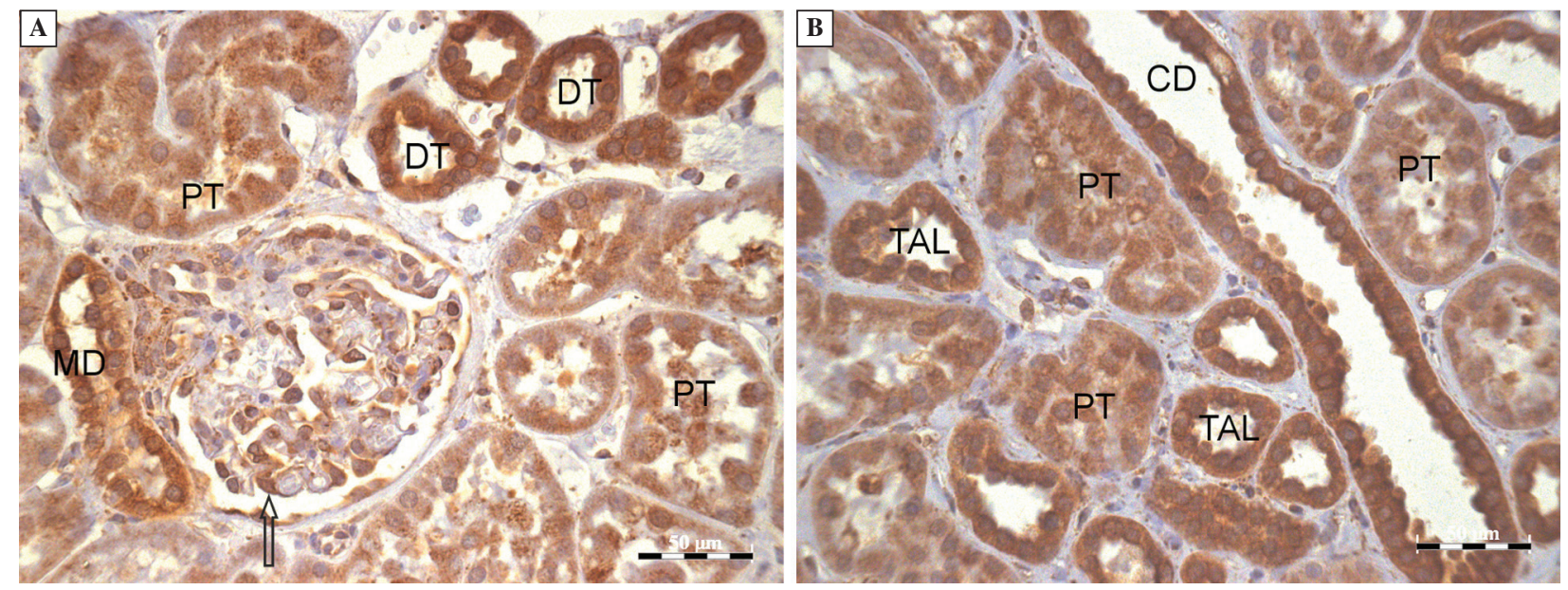

Figure 3. PLAGL1 distribution in the cortex (A) and outer stripe of outer medulla (B) in normal human kidney. A. Strong PLAGL1 immunoreactivity was noted in distal tubules (DT) and macula densa (MD). Less intense PLAGL1 immunostaining was present in proximal tubules (PT), podocytes (arrow) and parietal epithelium of Bowman's capsule; B. Moderate PLAGL1 immunoreactivity in proximal tubules (PT) and strong immunostaining in thick ascending limbs of Henle's loop (TAL) and collecting duct (CD). IHC staining was performed as described in Methods. Total magnification $\times 400$; scale bar, $50 \mu \mathrm{m}$

noted in endothelial cells of blood vessels (Figure 4). PLAGL1 immunoreactivity was localized mainly in the cytoplasm but it was also present in the cell nuclei, although nuclear expression of PLAGL1 was less ubiquitous and weaker than the cytoplasmic. Strong PLAGL1 staining intensity levels were reported in the epithelial cells of distal tubules and collecting ducts (scored for $45.4 \pm 3.9 \%$ and $48.7 \pm 1.8 \%$, mean $\pm \mathrm{SD}$, respectively; Figure 5). They were significantly higher than moderate or weak PLAGL1 expression noted in proximal tubules and renal corpuscles $(21.8 \pm$ $3.1 \%$ and $13.6 \pm 2.3 \%$, respectively; Figure 5). PLAGL1 immunoreactivity level in thick ascending limbs of Henle's loop was similar to those revealed in distal tubules and collecting ducts and was not included into statistical analysis. No correlations 

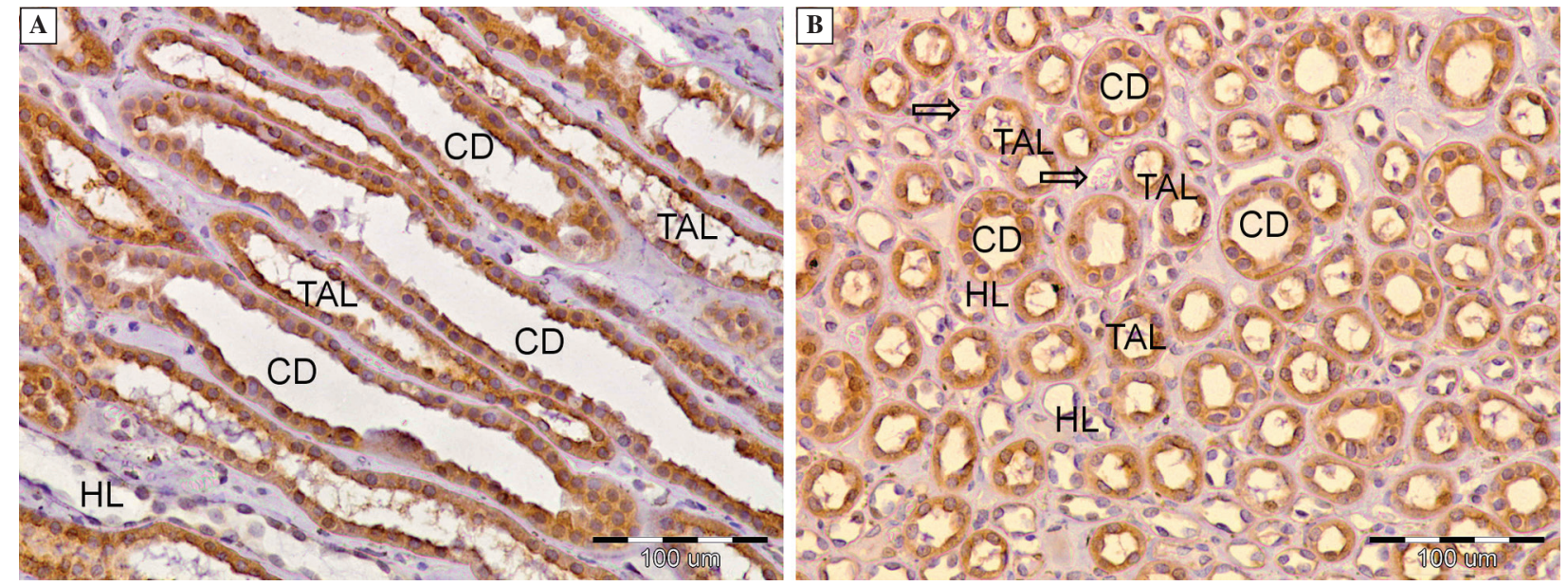

Figure 4. PLAGL1 distribution in the inner medulla of normal human kidney in longitudinal (A) and cross (B) sections. A. Strong immunoreactivity was noted in the cytoplasm and nuclei of collecting duct (CD) and thick ascending limbs of Henle's loop (TAL); B. Weak immunoreactivity was found in some but not all nuclei of thin limbs of Henle's loop cells (HL). No reaction was found in blood vessels' (arrows) endothelial cells. IHC staining was performed as described in Methods. Total magnification $\times 200$; scale bar, $100 \mu \mathrm{m}$

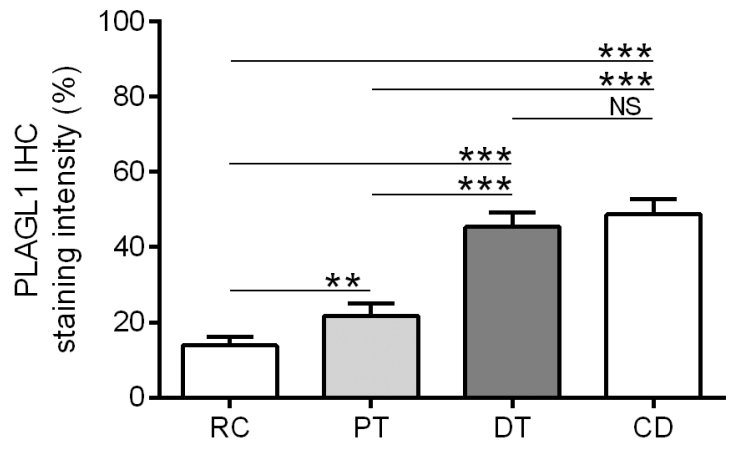

Figure 5. Semi-quantitative assessment of the intensity of PLAGL1 immunoreactivity in different kidney compartments. RC — renal corpuscles; PT — proximal tubules; DT — distal tubules; CD — collecting ducts. ${ }^{* *},{ }^{* * *}$ statistically significant differences in staining intensity noted between indicated segments of uriniferous tubule, $p<0.01$ and $p<0.001$, respectively; NS - not significant $(p>0.05)$

were disclosed between the PLAGL1 immunoreactivity levels and clinicopathological data of patients (data not shown).

\section{Putative PLAGL1-regulated genes and their functional annotations}

A database of TSS-upstream sequences of 478 genes related with kidney physiology was sought for the presence of PLAGL-1 binding motifs. Putative cis-regulatory elements were identified in the 2-kb-upstre- am regions of 67 transcripts of 43 genes belonging to different functional groups including, transmembrane transport, metabolic processes, intracellular signaling and kidney development. Nineteen genes overlapped with at least one of KEGG's kidney-related pathways. A list of putative PLAGL1 target genes with selected GO and KEGG annotations is shown in Table 1. Analysis of functional annotations in the set of putative PLAGL1 target genes identified three significantly (corrected $p<0.05$ ) over-represented gene sets: inositol phosphate metabolic processes (GO), endocrine and other factor-regulated calcium reabsorption (KEGG) and calcium signaling pathways (KEGG) (Table 2).

\section{Discussion}

Earlier studies reported the presence of PLAGL1 in human kidney at the mRNA and protein levels by Northern [1] and Western blotting [18] techniques, respectively. However, the exact tissue-specific localization of PLAGL1 protein in human kidney was not determined to date. To the best of our knowledge, our study is the first report describing the expression of PLAGL1 in the structures of cortex and medulla of normal human kidney. We demonstrated that PLAGL1 staining intensity differed depending on the tubule type with the highest levels of PLAGL1 protein expression in the thick ascending limbs of Henle's loop, distal tubules and collecting ducts as opposed to renal corpuscles and proximal tubules, in which the immunoreactivity was weak or moderate. 
Table 1. Genes containing the core of putative PLAGL1-binding motif GGGGCCCC in their 2-kb-upstream sequence and overlapping kidney-related pathways (KEGG) and gene ontology

\begin{tabular}{|c|c|c|c|}
\hline HGNC symbol & Gene name & Gene ontology & KEGG \\
\hline AGMAT & Agmatine ureohydrolase (agmatinase) & D G H Q & \\
\hline ASS1 & Argininosuccinate synthase 1 & D F G H I J Q R S & \\
\hline ATP1B2 & ATPase, $\mathrm{Na}^{+} / \mathrm{K}^{+}$transporting, beta 2 polypeptide & A B D E P Q & 124 \\
\hline ATP2B1 & ATPase, $\mathrm{Ca}^{++}$transporting, plasma membrane 1 & A B E & 2 \\
\hline ATP6V1B1 & ATPase, $\mathrm{H}^{+}$transporting, lysosomal 56/58kDa, V1 subunit B1 & A B D E F J L P Q R S & 5 \\
\hline BHMT2 & Betaine-homocysteine S-methyltransferase 2 & D G H I O Q & \\
\hline COL4A3 & Collagen, type IV, alpha 3 (Goodpasture antigen) & $\mathrm{N}$ & \\
\hline DNM1 & Dynamin 1 & $\mathrm{E}$ & 2 \\
\hline DPEP1 & Dipeptidase 1 (renal) & D F G H I O Q S & \\
\hline DUSP15 & Dual specificity phosphatase 15 & P R & \\
\hline DYNC1LI1 & Dynein, cytoplasmic 1 , light intermediate chain 1 & $\mathrm{E}$ & 3 \\
\hline FGF9 & Fibroblast growth factor 9 & E F J P R S & \\
\hline FOXI2 & Forkhead box I2 & & \\
\hline FXYD2 & FXYD domain containing ion transport regulator 2 & A B E & 124 \\
\hline GALNT9 & Polypeptide $\mathrm{N}$-acetylgalactosaminyltransferase 9 & & \\
\hline GLS & Glutaminase & A D E GHQ & 4 \\
\hline GNAQ & Guanine nucleotide binding protein ( $\mathrm{G}$ protein), q polypeptide & D E P Q & 2 \\
\hline HRSP12 & Heat-responsive protein 12 & & \\
\hline INSRR & Insulin receptor-related receptor & $\mathrm{P}$ & \\
\hline KL & Klotho & F J P R S & 2 \\
\hline MFSD4 & Major facilitator superfamily domain containing 4 & $\mathrm{~B}$ E & \\
\hline PDPK1 & 3-phosphoinositide dependent protein kinase 1 & A EF J P R S & 1 \\
\hline PIPOX & Pipecolic acid oxidase & D G H I O P Q & \\
\hline PLCB2 & Phospholipase C, beta 2 & C D M P & 2 \\
\hline PLCB3 & Phospholipase C, beta 3 (phosphatidylinositol-specific) & C D M P & 2 \\
\hline PLCB4 & Phospholipase C, beta 4 & C D M P & 2 \\
\hline PNPLA1 & Patatin-like phospholipase domain containing 1 & & \\
\hline PODXL & Podocalyxin-like & $\mathrm{N}$ & \\
\hline PTH1R & Parathyroid hormone 1 receptor & C D M P & 2 \\
\hline RAB11FIP3 & RAB11 family interacting protein 3 (class II) & $\mathrm{E}$ & \\
\hline RAB5C & RAB5C, member RAS oncogene family & D E P Q & 3 \\
\hline RDH8 & Retinol dehydrogenase 8 (all-trans) & $\mathrm{DM}$ & \\
\hline RENBP & Renin binding protein & $\mathrm{DG}$ & \\
\hline RNF152 & Ring finger protein 152 & & \\
\hline SGK2 & Serum/glucocorticoid regulated kinase 2 & A B E P & \\
\hline SLC12A3 & Solute carrier family 12 (sodium/chloride transporter), member 3 & A B E & \\
\hline SLC13A1 & Solute carrier family 13 (sodium/sulfate symporter), member 1 & A B E K & \\
\hline SLC22A8 & Solute carrier family 22 (organic anion transporter), member 8 & A B E F J KS & \\
\hline SLC25A10 & $\begin{array}{l}\text { Solute carrier family } 25 \text { (mitochondrial carrier; dicarboxylate transporter), } \\
\text { member } 10\end{array}$ & A B D E G H O Q & 4 \\
\hline SLC2A1 & Solute carrier family 2 (facilitated glucose transporter), member 1 & A B D E G & 6 \\
\hline SLC2A9 & Solute carrier family 2 (facilitated glucose transporter), member 9 & A B D E G L Q & \\
\hline SLC8A1 & Solute carrier family 8 (sodium/calcium exchanger), member 1 & A B E F J R S & 2 \\
\hline SLC9A3* & Solute carrier family 9, subfamily A (NHE3, cation proton antiporter 3), member 3 & A B E L & 4 \\
\hline
\end{tabular}

GO biological processes: A - ion transport GO:0006811; B — transmembrane transport GO:0055085; C - inositol phosphate metabolic process GO:0043647; D — small molecule metabolic process GO:0044281; E — transport GO:0006810; F — response to nitrogen compound GO:1901698; G - organic acid metabolic process GO:0006082; H — cellular amino acid metabolic process GO:0006520; I — cellular modified amino acid metabolic process GO:0006575; J — response to organonitrogen compound GO:0010243; K — sulfur compound transport GO:0072348; L — hydrogen transport GO:0006818; M — organic hydroxy compound metabolic process GO:1901615; N — glomerulus development GO:0032835; O — sulfur compound metabolic process GO:0006790; P — phosphorus metabolic process GO:0006793; Q — organonitrogen compound metabolic process GO:1901564; R — cellular response to endogenous stimulus GO:0071495; S — response to oxygen-containing compound GO:1901700

KEGG pathways: 1 - aldosterone-regulated sodium reabsorption hsa04960; 2 - endocrine and other factor-regulated calcium reabsorption hsa04961; 3 - vasopressin-regulated water reabsorption hsa04962;4 - proximal tubule bicarbonate reclamation hsa04964;5 - collecting duct acid secretion hsa04966; 6 - renal cell carcinoma hsa05211 
Table 2. Over-represented gene ontology annotation sets (GO) and pathways (KEGG) of the genes containing putative PLAGL1-binding motif(s) GGGGCCCC in their 2-kb-upstream sequence

\begin{tabular}{|l|l|l|c|c|c|c|}
\hline Over-represented gene-sets & Source & Overlapping genes & Count & $\begin{array}{c}\text { Fold } \\
\boldsymbol{p} \text {-value }\end{array}$ & $\begin{array}{c}\text { Corrected } \\
\boldsymbol{p} \text {-value }\end{array}$ \\
\hline $\begin{array}{l}\text { Inositol phosphate metabolic } \\
\text { process }\end{array}$ & $\begin{array}{l}\text { BP level 3 } \\
\text { GO:0043647 }\end{array}$ & PTH1R; PLCB2; PLCB3; PLCB4 & 4 & 8.78 & 0.0003 & 0.0434 \\
\hline $\begin{array}{l}\text { Endocrine and other factor- } \\
\text {-regulated calcium reab- } \\
\text { sorption (Homo sapiens) }\end{array}$ & KEGG: hsa04961 & $\begin{array}{l}\text { PTH1R, PLCB2, PLCB3, PLCB4, } \\
\text { GNAQ, ATP1B2, KL, ATP2B1, } \\
\text { DNM1, SLC8A1, FXYD2 }\end{array}$ & 11 & 2.60 & 0.0011 & 0.0200 \\
\hline $\begin{array}{l}\text { Calcium signaling pathway } \\
- \text { Homo sapiens (human) }\end{array}$ & KEGG: hsa04020 & $\begin{array}{l}\text { PLCB2; PLCB3; PLCB4; GNAQ; } \\
\text { ATP2B1; SLC8A1 }\end{array}$ & 6 & 2.98 & 0.0094 & 0.0332 \\
\hline
\end{tabular}

Count — number of members of predefined gene-set (GO or KEGG) in user-specified gene set; Fold — ratio between observed (enriched) and expected (background corrected) incidence of members of predefined gene-set (GO or KEGG) in user-specified gene set; $p$-values and corrected $p$-values - are calculated according to the hypergeometric test and then corrected for multiple testing using the false discovery rate method as described in http://cpdb.molgen.mpg.de/ [17]

For each patient included in this study the lack of lesions in the analyzed kidney section was confirmed by $\mathrm{H} \&$ E staining. The normal morphology of kidney tissue can be also supported by the fact that the levels of PLAGL1 immunoreactivity did not correlate with clinicopathological data of patients (data not shown). Additionally, by the Western blotting technique we confirmed that the antibodies used in our IHC study specifically bind to a $51 \mathrm{kDa}$-protein in homogenates obtained from normal human kidney. The molecular mass of detected band corresponds well to the one of PLAGL1 isoform 2 (50.8 kDa). Our morphological and biochemical data are in concordance with biochemical study which showed strong level of PLAGL1 protein expression in the homogenates of human kidney papilla and medulla with weak expression in the kidney cortex [18].

It has been established that the epithelial cells of any given segment of the uriniferous tubule [divided according to the current morphological nomenclature into the proximal tubule, the intermediate (thin) tubule, the distal tubule and the collecting duct] are homogeneous and distinct for that segment, and that structural differences between them reflect the localization and function of transport proteins and receptors [19]. The different immunoreactivity of the PLAGL1 protein in the various parts of nephron and collecting tubules demonstrated in our study may express its involvement in specific transepithelial transport processes. However, due to the multiplicity of the luminal and basolateral transport proteins we can implicate only some of them in the context of PLAGL1 distribution in normal human kidney. Therefore we hypothesize that differences in PLAGL1 immunostaining could reflect the involvement of PLAGL1 in the process of nuclear receptor-regulated expression of proteins such as $\mathrm{Na}^{+} / \mathrm{K}^{+}$-ATPase subunits. PLAGL1 mRNA expression was found in the human kidney and others tissues which are regulated by steroid hormones as compared to non -steroid dependent other organs and tissues [1]. It was documented that PLAGL1 protein may directly and indirectly co-activate the nuclear receptors [12]. Furthermore, the Human Protein Atlas documentation of human kidney immunostained against the mineralocorticoid receptor (NR3C2) protein showed a similar pattern of staining distribution as PLAGL1 found in our study, i.e. the cytoplasmic staining of epithelial cells was visible in all nephron tubules with a stronger immunoreactivity in the distal tubules (Human Protein Atlas, http://www.proteinatlas.org/ /ENSG00000151623-NR3C2/tissue/kidney) [20]. Such mechanisms of PLAGL1 action strongly suggest its potential role in kidney as a factor contributing to the renal aldosterone-regulated processes.

Other studies also report that PLAGL1 may affect kidney water homeostasis. The water-restricted mice, i.e. subjected to hyperosmotic condition, had increased levels of PLAGL1 mRNA as well as PLAGL1 protein in the kidney papilla (assessed by Western blotting) as opposed to lower expression of both mRNA and protein in the kidney cortex. Similarly, much lower PLAGL1 protein levels were found in normal human kidney in cortex than in medulla and papilla [18]. The demonstration that PLAGL1 is up-regulated under hypertonic stress and negatively regulates expression of the sorbitol dehydrogenase suggests that this protein may be involved in the organic osmolyte regulation in mammalian kidney [18].

Considering the alternative roles of PLAGL1 in kidney, we assumed that its transcriptional activity can be mediated in two ways: directly by DNA-binding and indirectly by modulating the activity of other transcription factors. Since PLAGL1 can directly 
regulate gene transcription by sequence-specific DNA binding in the promoter region of target genes $[1,8]$ we sought for putative PLAGL1-regulated genes among the group of genes associated with kidney physiology. By in sillico screening of promoter sequences for alignment with the core of PLAGL1 DNA-binding site GGGGCCCC we designated 43 candidate genes for PLAGL1-regulated genes. The set of putative PLAGL1-target genes was analyzed for functional annotations revealing gene ontology and associations with kidney-related pathways (Table 1). The PLAGL1-target genes were found to be associated mainly with transport, including SLC- and ATPase-mediated membrane transport, phosphate metabolism including Gq/PLC-beta signaling pathway and glomerulus filtration barrier components (Table 2). Bioinformatics analysis revealed that putative PLAGL1-binding motifs are over-represented in the set of genes involved in the regulated calcium reabsorption and calcium signaling (Tables 1 and 2). Hypothetical PLAGL1 targets included also genes coding for membrane transporters and ATPases, members of the inositol phosphate pathway, klotho glucuronidase, dynamin and parathyroid receptor 1 (Table 1). Since PLAGL1 can act as a transcriptional cofactor of various nuclear receptors [12], the interaction of PLAGL1 with vitamin D, estrogen or androgen receptors cannot be excluded. Consequently, we hypothesize that PLAGL1 could be a factor which can both directly, by DNA-binding and transactivation, or indirectly, regulate or modulate the transcriptional activity of mineralocorticoid and glucocorticoid receptors [12]. The hypothetical involvement of PLAGL1 in the control of both calcium reabsorption and aldosterone-regulated sodium-potassium transport can be supported by the fact that segments of the uriniferous tubule known as localization for these processes [21, 22] overlap with the pattern of PLAGL1 expression observed in the present study. It was also documented that aldosterone-activated mineralocorticoid receptors can influence the expression of sodium bicarbonate transporters SLC9A3 and $\mathrm{Na}^{+}$/ $/ \mathrm{K}^{+}$-ATPase subunit alpha in the epithelial cells of proximal tubule [23]. PLAGL1-binding sequences were found in the promoter of $S L C 2 A 1$ gene encoding for GLUT1 transporter which is present in the basolateral membrane of the proximal tubule epithelial cells [24]. The expression of $S L C 2 A 1$ is regulated by the VHL/HIF1 pathway and associated with the progression of renal cell carcinoma [25], indicating another hypothetical role of PLAGL1 transcriptional activity under pathological conditions. The presence of PLAGL1 protein in the renal corpuscles could be also associated with the regulation of the expression
PODXL and COL $4 A 3$ genes which protein products, podocalyxin and collagen type IV, play a key role in the maintaining and functioning of the renal filtration barrier [26, 27]. Taken together, these findings could explain the ubiquitous renal immunoreactivity of the PLAGL1 protein observed in the present study.

In conclusion, we report experimental data documenting a widespread but quantitatively differentiated immunoexpression of the PLAGL1 protein in normal human kidney. We hypothesize that PLAGL1 can regulate expression of numerous genes by acting as a transcription factor and/or transcriptional coactivator/ /corepressor of nuclear receptors. These two modes of PLAGL1 action can overlap, showing a potency of PLAGL1 to integrate and synergize several regulatory pathways by the way of its DNA-binding and protein-binding activities. The exact role of PLAGL1 in the physiology (and pathology) of kidney cannot be elucidated without further studies.

\section{Acknowledgment}

This study was supported by the National Science Center grant No NN 402452839.

\section{References}

1. Varrault A, Ciani E, Apiou F et al. hZAC encodes a zinc finger protein with antiproliferative properties and maps to a chromosomal region frequently lost in cancer.Proc Natl Acad Sci USA. 1998;95:8835-8840. doi: 10.1073/pnas.95.15.8835.

2. Abdollahi A. LOT1 (ZAC1/PLAGL1) and its family members: mechanisms and functions. J Cell Physiol. 2007;210:16-25. doi: $10.1002 /$ jcp. 20835 .

3. Varrault A, Gueydan C, Delalbre A, Bellmann A, Houssami S. Zac1 regulates an imprinted gene network critically involved in the control of embryonic growth. Dev. Cell. 2006;11:711-722. doi: 10.1016/j.devcel.2006.09.003.

4. Mackay DJ, Coupe AM, Shield JP, Storr JN, Temple IK, Robinson DO. Relaxation of imprinted expression of ZAC and HYMAI in a patient with transient neonatal diabetes mellitus. Hum Genet. 2002;110:139-144. doi: 10.1007/s00439$-001-0671-5$.

5. Kamiya M, Judson H, Okazaki Y et al. The cell cycle control gene ZAC/PLAGL1 is imprinted - a strong candidate gene for transient neonatal diabetes. Hum Mol Genet. 2000;9:453-460. doi: $10.1093 / \mathrm{hmg} / 9.3 .453$.

6. Spengler D, Villalba M, Hoffmann A, Pantaloni C. Regulation of apoptosis and cell cycle arrest by Zac1, a novel zinc finger protein expressed in the pituitary gland and the brain. EMBO J. 1997;16:2814-2825. doi: 10.1093/emboj/16.10.2814.

7. Valente T, Auladell C. Expression pattern of Zac1 mouse gene, a new zinc-finger protein that regulates apoptosis and cellular cycle arrest, in both adult brain and along development. Mech Dev. 2001;108:207-211. doi: 10:1016/S0925-4773(01)00492-0.

8. Hensen K, Van Valckenborgh IC, Kas K, Van de Ven WJ, Voz ML. The tumorigenic diversity of the three PLAG family members is associated with different DNA binding capacities. Cancer Res. 2002;62:1510-1517. PMID: 11888928. 
9. Hoffmann A, Barz T, Spengler D. Multitasking C2H2 zinc fingers link Zac DNA binding to coordinated regulation of p300-histone acetyltransferase activity. Mol Cell Biol. 2006;26:5544-5557. doi: 10.1128/MCB.02270-05.

10. Hoffmann A, Spengler D. A new coactivator function for Zac1's C2H2 zinc finger DNA-binding domain in selectively controlling PCAF activity. Mol Cell Biol. 2008;28:6078-6093. doi: 10.1128/MCB.00842-08.

11. Huang SM, Schönthal AH, Stallcup MR. Enhancement of p53-dependent gene activation by the transcriptional coactivator Zac1. Oncogene. 2001;20:2134-2143. doi: 10.1038/ /sj.onc.1204298.

12. Huang SM, Stallcup MR. Mouse Zac1, a transcriptional coactivator and repressor for nuclear receptors. Mol Cell Biol. 2000;20:1855-1867. doi: 10.1128/MCB.20.5.1855-1867.2000.

13. Kowalczyk AE, Godlewski J, Krazinski BE et al. Divergent expression patterns of SATB1 mRNA and SATB1 protein in colorectal cancer and normal tissues. Tumor Biology. 2015. doi: 10.1007/s13277-015-3084-0.

14. Kasprzyk A. BioMart: driving a paradigm change in biological data management. Database (Oxford). 2011;2011:bar049. doi: 10.1093/database/bar049.

15. Kanehisa M, Goto S, Sato Y, Kawashima M, Furumichi M, Tanabe M. Data, information, knowledge and principle: back to metabolism in KEGG. Nucleic Acids Res. 2014;42(Database issue):D199-D205. doi: 10.1093/nar/gkt1076.

16. Uhlén M, Fagerberg L, Hallström BM et al. Proteomics. Tissue-based map of the human proteome. Science. 2015;347: 1260419. doi: 10.1126/science.1260419.

17. Kamburov A, Stelzl U, Lehrach H, Herwig R. The ConsensusPathDB interaction database: 2013 update. Nucleic Acids Res. 2013;41(Database issue):D793-D800. doi: 10.1093/nar/gks1055.

18. Lanaspa MA, Andres-Hernando A, Rivard CJ, Dai Y, Li N, Berl T. ZAC1 is up-regulated by hypertonicity and decreases sorbitol dehydrogenase expression, allowing accumulation of sorbitol in kidney cells. J Biol Chem. 2009;284:19974-19981. doi: 10.1074/jbc.M109.001792.

19. Christensen EI, Wagner CA, Kaissling B. Uriniferous tubule: structural and functional organization. Compr Physiol. 2012;2:805-861. doi: 10.1002/cphy.c100073.

20. Uhlen M, Oksvold P, Fagerberg L et al. Towards a knowledge-based Human Protein Atlas. Nat Biotechnol. 2010;28:1248-1250. doi: 10.1038/nbt1210-1248.

21. Dimke H, Hoenderop JG, Bindels RJ. Hereditary tubular transport disorders: implications for renal handling of $\mathrm{Ca} 2+$ and Mg2+. Clin Sci (Lond). 2009;118:1-18. doi: 10.1042/ /CS20090086.

22. Meneton P, Loffing J, Warnock DG. Sodium and potassium handling by the aldosterone-sensitive distal nephron: the pivotal role of the distal and connecting tubule. $\mathrm{Am}$ J Physiol Renal Physiol. 2004;287:F593-F601. doi: 10.1152/ /ajprenal.00454.2003.

23. Salyer SA, Parks J, Barati MT et al. Aldosterone regulates $\mathrm{Na}(+), \mathrm{K}(+)$ ATPase activity in human renal proximal tubule cells through mineralocorticoid receptor. Biochim Biophys Acta. 2013;1833:2143-2152. doi: 10.1016/j.bbamcr. 2013.05.009.

24. Mitrakou A. Kidney: its impact on glucose homeostasis and hormonal regulation. Diabetes Res Clin Pract. 2011;Suppl 1: S66-S72. doi: 10.1016/S0168-8227(11)70016-X.

25. Clark PE. The role of VHL in clear-cell renal cell carcinoma and its relation to targeted therapy. Kidney Int. 2009;76:939-945. doi: 10.1038/ki.2009.296.

26. Nielsen JS, McNagny KM. The role of podocalyxin in health and disease. J Am Soc Nephrol. 2009;20:1669-1676. doi: 10.1681/ASN.2008070782.

27. Abrahamson DR, Hudson BG, Stroganova L, Borza DB, St John PL. Cellular origins of type IV collagen networks in developing glomeruli. J Am Soc Nephrol. 2009;20:1471-1479. doi: 10.1681/ASN.2008101086.

Submitted: 26 March, 2015

Accepted after reviews: 30 March, 2015 Available as AoP: 31 March, 2015 